\title{
A DAMPED TELEGRAPH RANDOM PROCESS WITH LOGISTIC STATIONARY DISTRIBUTION
}

\author{
ANTONIO DI CRESCENZO $* * *$ AND \\ BARBARA MARTINUCCI, ${ }^{* * *}$ Università degli Studi di Salerno
}

\begin{abstract}
We introduce a stochastic process that describes a finite-velocity damped motion on the real line. Differently from the telegraph process, the random times between consecutive velocity changes have exponential distribution with linearly increasing parameters. We obtain the probability law of the motion, which admits a logistic stationary limit in a special case. Various results on the distributions of the maximum of the process and of the first passage time through a constant boundary are also given.
\end{abstract}

Keywords: Alternating random process; logistic stationary distribution; distribution of the maximum; first passage time

2000 Mathematics Subject Classification: Primary 60K15

Secondary 60K99

\section{Introduction}

The (integrated) telegraph process has been studied in the past by many authors aiming to describe a random motion at constant speed on the real line, whose velocity alternates according to a Poisson process. Problems related to this process, such as absorption and first passage times, have been treated, for instance, by Foong [6] and Orsingher [16], [17]. Restricting our attention to one-dimensional models, various kinds of generalizations of the telegraph process have been proposed in the literature towards

- motions characterized by more than two velocities (see [9] and [18]) or by random velocities (see [22]);

- velocity changes governed by an inhomogeneous Poisson process (see [8]) or by an alternating renewal process (see [24]);

- velocities alternating at gamma- or Erlang-distributed random times (see [2], [3], and [19]);

- exponential transformations leading to market models (see [4] and [21]).

However, the explicit law of the resulting processes has been obtained in few cases.

In this paper we aim to modify anew the telegraph process by assuming that the random times separating consecutive velocity changes are still exponentially distributed, but with linearly increasing parameters $\lambda k$ and $\mu k$. The resulting process $X_{t}$ describes a random motion with two

Received 20 May 2009; revision received 17 December 2009.

* Postal address: Dipartimento di Matematica e Informatica, Università degli Studi di Salerno, Via Ponte don Melillo, I-84084 Fisciano (SA), Italy.

This work has been partially supported by Regione Campania and MIUR (PRIN 2008).

** Email address: adicrescenzo@unisa.it

*** Email address: bmartinucci@unisa.it 
velocities, $c$ and $-v$, that alternate at stochastically decreasing times. This is a kind of damped motion, in the sense that the continuous sample paths of $X_{t}$ are composed of line segments that become stochastically smaller and smaller. The idea of a 'damped telegraph random process' seems to be new in the literature, whereas in the realm of probability theory can be found several works dealing with damped diffusion processes. These are based on the modification of the infinitesimal moments of a continuous-time diffusion process by introduction of a damping function. Damped diffusion processes have been applied in a broad class of financial models, such as models of stock prices. For instance, the case of a flexible process able to break many types of asset price bubbles and preserve the martingale pricing approach is proposed in [11]. Damped diffusion processes have also been used in the physics literature, for instance, to describe inertial motions driven by dichotomous Markov noise in the absence of a potential [13].

General damped processes are frequently exploited to describe the random evolution of physical systems. In particular, damped telegraph random processes can model damping behavior appearing in particle systems characterized by finite velocities. We recall that the usefulness of finite-velocity random motions emerges in various applied fields, such as (i) singleserver queueing systems characterized by queueing room of size 1 (see Application 1.1 of [2], and [5]) and multi-files queueing systems (see Section 2 of [10]); (ii) alternating renewal processes governing repairable systems in reliability theory (see Application 1.2 of [2]); and (iii) stochastic processes in mathematical finance with alternating growth rates, such as the geometric telegrapher's process [4].

In Section 2 we formally define the process $X_{t}$, and give some preliminary results on the transition densities in the more general case when the random times between consecutive changes of velocities have general distributions. In Section 3 we introduce the case when such random times have exponential distribution with linear parameters. In this framework we obtain the probability law of $X_{t}$, which is characterized by a discrete component on the extremes of the domain $[-v t, c t]$, and by an absolutely continuous component over its interior. We discuss the limit behavior of the probability density of $X_{t}$. The specific assumption of stochastically decreasing switching times allows the existence of a stationary distribution. Indeed, differently from other finite-velocity random motions, in a special case of our model the transition density of $X_{t}$ tends to a logistic density as time diverges. Such stationary density emerges when the ratios between the parameters characterizing the forward and the backward motions are equal (i.e. $\lambda / c=\mu / v)$. We also obtain the mean of $X_{t}$ (when $\lambda=\mu$ ) conditional on the initial velocity. In Section 4 we deal with the maximum of $X_{t}$. We develop a procedure based on an iterative definition of nested integrals of distribution functions, which allows us to obtain (when $\lambda / c=\mu / v$ ) the distribution of the maximum of $X_{t}$, the first passage time distribution of $X_{t}$ through a fixed boundary $\beta$, and the corresponding first crossing time probability.

\section{A stochastic model of random motion}

We aim to study the motion on $\mathbb{R}$ of a particle starting at the origin, and moving with two velocities $c$ and $-v(c, v>0)$ that alternate randomly in time. The sign of the velocity determines at each instant the direction of motion (forward or backward). We denote by $U_{k}$ and $D_{k}$ the durations of the $k$ th time intervals during which the motion goes forward and backward, respectively, with $\left\{U_{k} ; k=1,2, \ldots\right\}$ and $\left\{D_{k} ; k=1,2, \ldots\right\}$ mutually independent sequences of independent nonnegative random variables. Differently from the cases treated in [2] and [3], the random variables of each sequence $\left\{U_{k}\right\}$ and $\left\{D_{k}\right\}$ are not necessarily identically distributed.

The random motion is described by a stochastic process $\left\{\left(X_{t}, V_{t}\right) ; t \geq 0\right\}$, having state space $\mathbb{R} \times\{-v, c\}$, where $X_{t}$ and $V_{t}$ respectively denote the position and velocity of the particle at 
time $t$. We assume that the motion starts from the origin, i.e. $X_{0}=0$, and that the initial velocity is random, with

$$
\mathrm{P}\left\{V_{0}=-v\right\}=\mathrm{P}\left\{V_{0}=c\right\}=\frac{1}{2} .
$$

Note that, for any fixed $t>0, X_{t}$ takes values in $[-v t, c t]$. Denoting by $T_{n}$ the $n$th random instant in which the motion changes velocity, we have

$$
T_{2 k}=U^{(k)}+D^{(k)}, \quad T_{2 k+1}=T_{2 k}+\left\{\begin{array}{ll}
U_{k+1} & \text { if } V_{0}=c, \\
D_{k+1} & \text { if } V_{0}=-v,
\end{array} \quad k=0,1, \ldots,\right.
$$

where $U^{(0)}=D^{(0)}=0$ and

$$
U^{(k)}=U_{1}+U_{2}+\cdots+U_{k}, \quad D^{(k)}=D_{1}+D_{2}+\cdots+D_{k}, \quad k=1,2, \ldots
$$

The position and velocity of the particle at time $t$ are expressed respectively as

$$
X_{t}=\int_{0}^{t} V_{s} \mathrm{~d} s, \quad V_{t}=\frac{c-v}{2}+\operatorname{sgn}\left(V_{0}\right) \frac{c+v}{2}(-1)^{N_{t}},
$$

where $\left\{N_{t} ; t \geq 0\right\}$ is the alternating counting process, characterized by random times $T_{1}, T_{2}, \ldots$, which counts the number of velocity reversals of the particle in $[0, t]$. In other terms, we have

$$
N_{0}=0, \quad N_{t}=\sum_{n=1}^{\infty} \mathbf{1}_{\left\{T_{n} \leq t\right\}}, \quad t>0 .
$$

Assuming that $U_{k}$ and $D_{k}$ are absolutely continuous random variables, the conditional probability law of $\left\{\left(X_{t}, V_{t}\right) ; t \geq 0\right\}$ possesses a discrete component

$$
\mathrm{P}\left\{X_{t}=y t, V_{t}=y \mid X_{0}=0, V_{0}=y\right\}
$$

and an absolutely continuous component

$$
p(x, t \mid y)=f(x, t \mid y)+b(x, t \mid y),
$$

where

$$
\begin{gathered}
f(x, t \mid y)=\frac{\partial}{\partial x} \mathrm{P}\left\{X_{t} \leq x, V_{t}=c \mid X_{0}=0, V_{0}=y\right\} \\
b(x, t \mid y)=\frac{\partial}{\partial x} \mathrm{P}\left\{X_{t} \leq x, V_{t}=-v \mid X_{0}=0, V_{0}=y\right\}
\end{gathered}
$$

with $t>0,-v t<x<c t$, and $y \in\{-v, c\}$.

Hereafter, we formally express the probability law of $\left\{\left(X_{t}, V_{t}\right) ; t \geq 0\right\}$ in terms of the density functions of (2.2), say $f_{U}^{(k)}$ and $f_{D}^{(k)}$, and of the survival functions of $U_{k}$ and $D_{k}$, denoted respectively as $\bar{F}_{U_{k}}$ and $\bar{F}_{D_{k}}$.

Proposition 2.1. Let $V_{0}=c$. For all $t>0$, we have

$$
\mathrm{P}\left\{X_{t}=c t, V_{t}=c \mid X_{0}=0, V_{0}=c\right\}=\bar{F}_{U_{1}}(t)
$$


moreover, for $-v t<x<c t$,

$$
\begin{array}{r}
f(x, t \mid c)=\frac{1}{c+v} \sum_{k=1}^{\infty} f_{D}^{(k)}\left(t-\tau_{*}\right) \int_{t-\tau_{*}}^{t} f_{U}^{(k)}\left(s-t+\tau_{*}\right) \bar{F}_{U_{k+1}}(t-s) \mathrm{d} s, \\
b(x, t \mid c)=\frac{1}{c+v} \sum_{k=0}^{\infty} f_{U}^{(k+1)}\left(\tau_{*}\right) \int_{\tau_{*}}^{t} f_{D}^{(k)}\left(s-\tau_{*}\right) \bar{F}_{D_{k+1}}(t-s) \mathrm{d} s,
\end{array}
$$

where $\tau_{*}=\tau_{*}(x, t)=(v t+x) /(c+v)$.

The proof proceeds similarly to that of Theorem 2.1 of [2] and is thus omitted. Furthermore, in analogy with Proposition 2.1 of [2] we have

$$
\mathrm{P}\left\{-v t \leq X_{t} \leq c t \mid X_{0}=0, V_{0}=c\right\}=1
$$

Remark 2.1. The probability law of $\left\{\left(X_{t}, V_{t}\right) ; t \geq 0\right\}$ conditional on $V_{0}=-v$ can be expressed as in Proposition 2.1 by interchanging $f$ with $b, U_{k}$ with $D_{k}$, $c$ with $v$, and $x$ with $-x$.

\section{Exponentially distributed intertimes with linear rates}

In the sequel we assume that the random times $U_{k}$ and $D_{k}$ separating consecutive velocity reversals of the particle have exponential distribution with parameters

$$
\lambda_{k}=\lambda k, \quad \mu_{k}=\mu k, \quad k=1,2, \ldots ; \lambda, \mu>0,
$$

respectively. Under this hypothesis, the reversal rates $\lambda_{k}$ and $\mu_{k}$ increase linearly with the number of reversals, so that the random intertimes $T_{2 k+1}-T_{2 k}$ and $T_{2 k}-T_{2 k-1}$ stochastically decrease with $k$. It follows that the sample paths of $X_{t}$ are subject to an increasing number of changes of velocity when $t$ increases, so that $X_{t}$ exhibits a damped motion. Figure 1 shows simulated sample paths of $X_{t}$ stopped after 200 changes of velocity.

The classical approach to finite-velocity random motions is based on the resolution of hyperbolic systems of partial derivative equations for the probability density of the process $\left\{\left(X_{t}, V_{t}\right) ; t \geq 0\right\}$. In this case we are led to define the following conditional densities, for
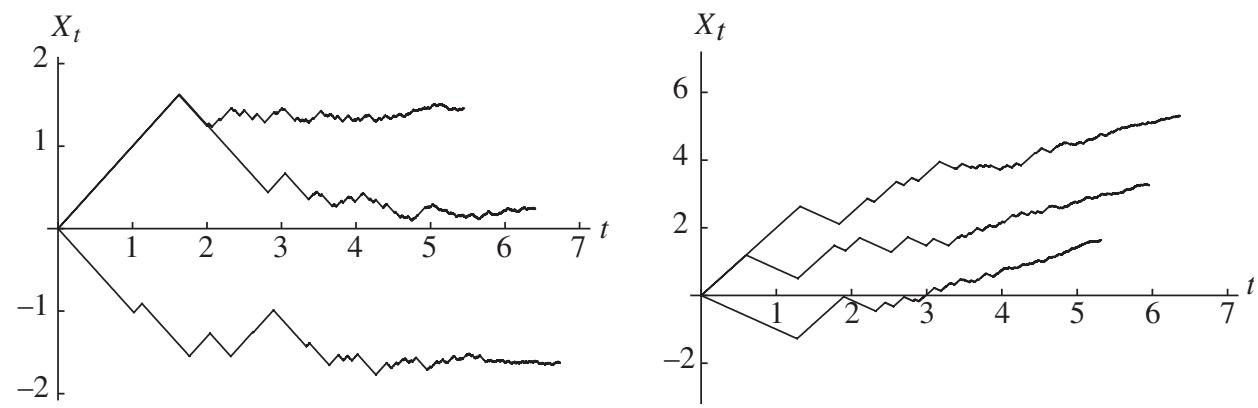

Figure 1: Simulated sample paths of $X_{t}$ for $\lambda=\mu=1$ and $v=1$, with $c=1$ (left) and $c=2$ (right). 


$$
\begin{aligned}
t>0,-v t<x<c t, & \text { and } n=1,2, \ldots: \\
f_{n}(x, t \mid c) & =\frac{\partial}{\partial x} \mathrm{P}\left\{X_{t} \leq x, V_{t}=c, T_{2 n-2} \leq t<T_{2 n-1} \mid X_{0}=0, V_{0}=c\right\}, \\
b_{n}(x, t \mid c) & =\frac{\partial}{\partial x} \mathrm{P}\left\{X_{t} \leq x, V_{t}=-v, T_{2 n-1} \leq t<T_{2 n} \mid X_{0}=0, V_{0}=c\right\}, \\
f_{n}(x, t \mid-v) & =\frac{\partial}{\partial x} \mathrm{P}\left\{X_{t} \leq x, V_{t}=c, T_{2 n-1} \leq t<T_{2 n} \mid X_{0}=0, V_{0}=-v\right\}, \\
b_{n}(x, t \mid-v) & =\frac{\partial}{\partial x} \mathrm{P}\left\{X_{t} \leq x, V_{t}=-v, T_{2 n-2} \leq t<T_{2 n-1} \mid X_{0}=0, V_{0}=-v\right\} .
\end{aligned}
$$

Making use of a customary procedure (see, for instance, [15]), it is not hard to see that the following system of differential equations holds:

$$
\begin{aligned}
\frac{\partial}{\partial t} f_{n}(x, t \mid c)= & -c \frac{\partial}{\partial x} f_{n}(x, t \mid c)-n \lambda f_{n}(x, t \mid c)+(n-1) \mu b_{n-1}(x, t \mid c), \quad n \geq 2, \\
\frac{\partial}{\partial t} b_{n}(x, t \mid c)= & v \frac{\partial}{\partial x} b_{n}(x, t \mid c)-n \mu b_{n}(x, t \mid c)+n \lambda f_{n}(x, t \mid c), \quad n \geq 1, \\
\frac{\partial}{\partial t} f_{n}(x, t \mid-v)= & -c \frac{\partial}{\partial x} f_{n}(x, t \mid-v)-n \lambda f_{n}(x, t \mid-v) \\
& +n \mu b_{n}(x, t \mid-v), \quad n \geq 1, \\
\frac{\partial}{\partial t} b_{n}(x, t \mid-v)= & v \frac{\partial}{\partial x} b_{n}(x, t \mid-v)-n \mu b_{n}(x, t \mid-v) \\
& +(n-1) \lambda f_{n-1}(x, t \mid-v), \quad n \geq 2 .
\end{aligned}
$$

Unfortunately, solving the above equations is a very hard task. In order to obtain the conditional probability law of $\left\{\left(X_{t}, V_{t}\right) ; t \geq 0\right\}$, we are thus led to pursue the approach based on Proposition 2.1. If $U_{k}$ and $D_{k}$ are exponentially distributed with parameters (3.1), the densities of $U^{(n)}$ and $D^{(n)}$ for $n \geq 1$ are given by

$$
\begin{array}{ll}
f_{U}^{(n)}(x)=n\left(1-\mathrm{e}^{-\lambda x}\right)^{n-1} \lambda \mathrm{e}^{-\lambda x}, & x>0, \\
f_{D}^{(n)}(x)=n\left(1-\mathrm{e}^{-\mu x}\right)^{n-1} \mu \mathrm{e}^{-\mu x}, & x>0 .
\end{array}
$$

Hence, $U^{(n)}$ and $D^{(n)}$ are distributed as the maximum of $n$ independent and identically distributed random variables having exponential distributions with parameters $\lambda$ and $\mu$, respectively. The formal proof follows from Lemma 1 of [23]. However, it can also be obtained by noting that, under assumption (3.1), $U_{k}$ and $D_{k}$ can be regarded as the spacings of random samples of independent random variables with exponential distributions, whose probability densities are given, for instance, in [20]. We recall that densities (3.2) and (3.3) belong to the generalized exponential class of distributions (see [7] and the references therein).

We are now able to obtain the probability law of $\left\{\left(X_{t}, V_{t}\right) ; t \geq 0\right\}$ conditional on $V_{0}=c$ when the random times $U_{k}$ and $D_{k}$ have exponential distributions with parameters (3.1).

Theorem 3.1. Let $U_{k}$ and $D_{k}$ be exponentially distributed with parameters $\lambda k$ and $\mu k, k \geq 1$, respectively. For all $t>0$, we have

$$
\mathrm{P}\left\{X_{t}=c t, V_{t}=c \mid X_{0}=0, V_{0}=c\right\}=\mathrm{e}^{-\lambda t}
$$


moreover, for $-v t<x<c t$,

$$
\begin{aligned}
f(x, t \mid c) & =\frac{\mu}{c+v} \frac{\mathrm{e}^{-\lambda \tau_{*}} \mathrm{e}^{-\mu\left(t-\tau_{*}\right)}\left(1-\mathrm{e}^{-\lambda \tau_{*}}\right)}{\left[\mathrm{e}^{-\lambda \tau_{*}}+\mathrm{e}^{-\mu\left(t-\tau_{*}\right)}\left(1-\mathrm{e}^{-\lambda \tau_{*}}\right)\right]^{2}}, \\
b(x, t \mid c) & =\frac{\lambda}{c+v} \frac{\mathrm{e}^{-\lambda \tau_{*}} \mathrm{e}^{-\mu\left(t-\tau_{*}\right)}}{\left[\mathrm{e}^{-\lambda \tau_{*}}+\mathrm{e}^{-\mu\left(t-\tau_{*}\right)}\left(1-\mathrm{e}^{-\lambda \tau_{*}}\right)\right]^{2}},
\end{aligned}
$$

where

$$
\tau_{*}=\tau_{*}(x, t)=\frac{v t+x}{c+v} .
$$

Proof. Since $U_{1}$ is exponentially distributed with parameter $\lambda$, (3.4) follows from (2.5). Furthermore, making use of (3.2) and (3.3), and recalling that $U_{k+1}$ is exponentially distributed with parameter $\lambda(k+1)$, from (2.6) we have

$$
\begin{aligned}
& f(x, t \mid c)= \frac{\mu}{c+v} \sum_{k=1}^{+\infty} k \mathrm{e}^{-\mu\left(t-\tau_{*}\right)}\left[1-\mathrm{e}^{-\mu\left(t-\tau_{*}\right)}\right]^{k-1} \\
& \quad \times \int_{t-\tau_{*}}^{t} \lambda k \mathrm{e}^{-\lambda\left(s-t+\tau_{*}\right)}\left[1-\mathrm{e}^{-\lambda\left(s-t+\tau_{*}\right)}\right]^{k-1} \mathrm{e}^{-\lambda(k+1)(t-s)} \mathrm{d} s \\
&=\frac{\mu}{c+v} \mathrm{e}^{-\mu\left(t-\tau_{*}\right)} \mathrm{e}^{-\lambda \tau_{*}}\left(1-\mathrm{e}^{-\lambda \tau_{*}}\right) \sum_{k=1}^{+\infty} k\left[1-\mathrm{e}^{-\mu\left(t-\tau_{*}\right)}\right]^{k-1}\left(1-\mathrm{e}^{-\lambda \tau_{*}}\right)^{k-1},
\end{aligned}
$$

which yields density (3.5). Equation (3.6) can be obtained similarly from (2.7).

Making use of (3.5)-(3.7) we now analyse the behavior of the probability density functions (PDFs) $f(x, t \mid c)$ and $b(x, t \mid c)$ when $x$ tends to the endpoints of the state space [-vt,ct].

Corollary 3.1. Under the assumptions of Theorem 3.1, we have

$$
\lim _{x \downarrow-v t} f(x, t \mid c)=0, \quad \lim _{x \uparrow c t} f(x, t \mid c)=\frac{\mu \mathrm{e}^{-\lambda t}}{c+v}\left(1-\mathrm{e}^{-\lambda t}\right),
$$

and

$$
\lim _{x \downarrow-v t} b(x, t \mid c)=\frac{\lambda \mathrm{e}^{-\mu t}}{c+v}, \quad \lim _{x \uparrow c t} b(x, t \mid c)=\frac{\lambda \mathrm{e}^{-\lambda t}}{c+v} .
$$

Note that the limits obtained in Corollary 3.1 are very close to those concerning the case when the random times $U_{k}$ and $D_{k}$ are identically distributed and have exponential distribution. (See Corollary 3.1 of [2] for $n=r=1$ ). This suggests that the effect of the velocity reversals on the PDFs (3.5) and (3.6) is more pronounced when $x$ is far from the endpoints of the interval $[-v t, c t]$.

Now it is not hard to obtain the probability law of $\left\{\left(X_{t}, V_{t}\right) ; t \geq 0\right\}$, which is characterized by a discrete component over points $c t$ and $-v t$, and by an absolutely continuous component on $(-v t, c t)$ with PDF

$$
p(x, t)=\frac{\partial}{\partial x} \mathrm{P}\left\{X_{t} \leq x \mid X_{0}=0\right\} .
$$

Theorem 3.2. Under the assumptions of Theorem 3.1, for all $t>0$, we have

$$
\begin{gathered}
\mathrm{P}\left\{X_{t}=c t, V_{t}=c \mid X_{0}=0\right\}=\frac{1}{2} \mathrm{e}^{-\lambda t}, \\
\mathrm{P}\left\{X_{t}=-v t, V_{t}=-v \mid X_{0}=0\right\}=\frac{1}{2} \mathrm{e}^{-\mu t} ;
\end{gathered}
$$



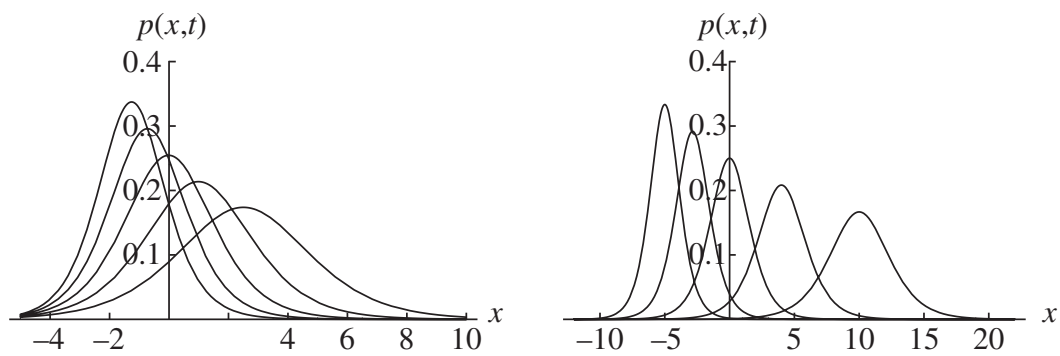

Figure 2: Densities $p(x, t)$ for $t=5$ (left) and $t=20$ (right) with $c=2, v=1, \mu=1$, and $\lambda=1,1.5,2,2.5,3$ (from right to left).

moreover, for $-v t<x<c t$,

$$
p(x, t)=\frac{\mathrm{e}^{-\lambda \tau_{*}} \mathrm{e}^{-\mu\left(t-\tau_{*}\right)}\left[2(\lambda+\mu)-\mu \mathrm{e}^{-\lambda \tau_{*}}-\lambda \mathrm{e}^{-\mu\left(t-\tau_{*}\right)}\right]}{2(c+v)\left[\mathrm{e}^{-\lambda \tau_{*}}+\mathrm{e}^{-\mu\left(t-\tau_{*}\right)}\left(1-\mathrm{e}^{-\lambda \tau_{*}}\right)\right]^{2}},
$$

where $\tau_{*}$ is defined in (3.7).

Proof. The proof immediately follows from (2.1), (2.3), (2.4), and (3.8), by recalling Theorem 3.1, and noting that the PDFs $f(x, t \mid-v)$ and $b(x, t \mid-v)$ can be obtained from (3.5) and (3.6) by the symmetry expressed in Remark 2.1.

Let us now evaluate the limits of density (3.9).

Corollary 3.2. Under the assumptions of Theorem 3.1, we have

$$
\begin{aligned}
\lim _{x \downarrow-v t} p(x, t) & =\frac{\mathrm{e}^{-\mu t}}{2(c+v)}\left[\mu+\lambda\left(2-\mathrm{e}^{-\mu t}\right)\right], \\
\lim _{x \uparrow c t} p(x, t) & =\frac{\mathrm{e}^{-\lambda t}}{2(c+v)}\left[\lambda+\mu\left(2-\mathrm{e}^{-\lambda t}\right)\right] .
\end{aligned}
$$

Plots of density (3.9) are given in Figure 2 for various choices of $\lambda$ and $t$.

Corollary 3.3. Under the assumptions of Theorem 3.1, if $\lambda v=\mu c$ then

$$
\lim _{t \rightarrow+\infty} p(x, t)=\frac{\theta}{4} \operatorname{sech}^{2}\left(\theta \frac{x}{2}\right), \quad x \in \mathbb{R},
$$

where

$$
\theta=\frac{\mu}{v}=\frac{\lambda}{c}
$$

if $\lambda v \neq \mu c$ then

$$
\lim _{t \rightarrow+\infty} p(x, t)=0, \quad x \in \mathbb{R} .
$$

Proof. Recalling (3.7) and (3.9), for $x \in \mathbb{R}$ and $t \rightarrow+\infty$, we have

$$
p(x, t) \sim \frac{1}{4} \frac{\lambda+\mu}{c+v} \operatorname{sech}^{2}\left(\frac{x(\lambda+\mu)+t(\lambda v-\mu c)}{2(c+v)}\right) .
$$

The proof then immediately follows. 


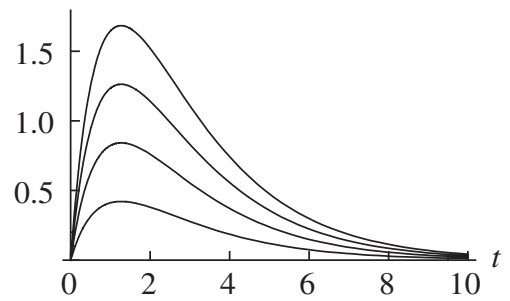

(a)

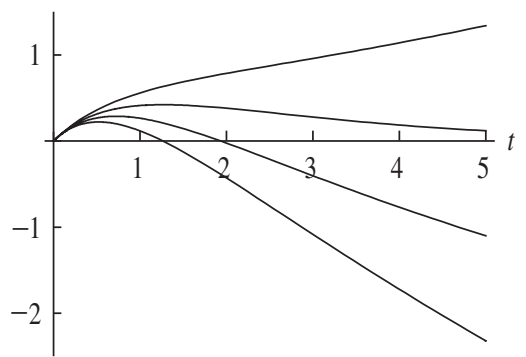

(b)

Figure 3: Plots of $\mathrm{E}\left[X_{t} \mid V_{0}=c\right]$ for (a) $\lambda=1$ and $c=v=1,2,3,4$ (from bottom to top) and (b) $c=1$ and $v=0.5,1,1.5,2$ (from top to bottom).

The right-hand side of (3.10) is a logistic density with mean 0 and variance $\pi^{2} /\left(3 \theta^{2}\right)$ (cf. [12] and [14] for recent characterization results on the logistic distribution).

Hereafter, we shall obtain the mean of $X_{t}$ conditional on the initial velocity in the special case when the random times $U_{k}$ and $D_{k}$ are identically exponentially distributed with parameter $\lambda_{k}=\lambda k$.

Theorem 3.3. Under the assumptions of Theorem 3.1, if $\lambda=\mu$ then, for all $t>0$, we have

$$
\mathrm{E}\left[X_{t} \mid V_{0}=c\right]=\frac{(c-v) t}{2}+\frac{c+v}{\lambda \sqrt{4 \mathrm{e}^{\lambda t}-1}} \arctan \left(\frac{\left(\mathrm{e}^{\lambda t}-1\right) \sqrt{4 \mathrm{e}^{\lambda t}-1}}{3 \mathrm{e}^{\lambda t}-1}\right) .
$$

Proof. Making use of (3.4)-(3.6) and by setting $y=(v t+x) /(c+v)$, we have

$$
\begin{array}{r}
\mathrm{E}\left[X_{t} \mid V_{0}=c\right]=c t \mathrm{e}^{-\lambda t}+\int_{0}^{t}[(c+v) y-v t] \frac{\mathrm{d}}{\mathrm{d} y}\left\{-\frac{\mathrm{e}^{-\lambda y}}{\mathrm{e}^{-\lambda y}+\mathrm{e}^{-\lambda(t-y)}\left(1-\mathrm{e}^{-\lambda y}\right)}\right\} \mathrm{d} y \\
=-v t+(c+v)\left\{\frac{t}{2}-\frac{1}{\lambda \sqrt{4 \mathrm{e}^{\lambda t}-1}}\left[\operatorname{arccot}\left(\sqrt{4 \mathrm{e}^{\lambda t}-1}\right)\right.\right. \\
\left.\left.+\arctan \left(\frac{1-2 \mathrm{e}^{\lambda t}}{\sqrt{4 \mathrm{e}^{\lambda t}-1}}\right)\right]\right\} .
\end{array}
$$

Hence, recalling Equations 4.4.36 and 4.4.16 of [1], (3.12) immediately follows.

The conditional mean given in (3.12) is shown in Figure 3 for $\lambda=1$ and some choices of the velocity.

Remark 3.1. Under the assumptions of Theorem 3.3, the mean of $X_{t}$ conditional on $V_{0}=-v$ can be easily obtained from (3.12), noting that, due to symmetry,

$$
\mathrm{E}\left[X_{t} \mid V_{0}=-v\right]+\mathrm{E}\left[X_{t} \mid V_{0}=c\right]=(c-v) t, \quad t>0 .
$$

\section{Distribution of the maximum}

A topic of interest within the field of random motions with finite velocities is the determination of the probability law of the maximum of the particle's position. We recall, for instance, the papers by Orsingher [16] and Foong [6] concerning the telegraph random process. 
Let us now introduce the distribution function of the maximum of the damped motion process $X_{t}$ conditional on initial velocity:

$$
F(\beta, t \mid y)=\mathrm{P}\left\{\max _{0 \leq s \leq t} X_{s}<\beta \mid X_{0}=0, V_{0}=y\right\}, \quad t \geq 0,
$$

with $0<\beta<c t$ and $y \in\{-v, c\}$. Aiming to obtain a closed-form result for $F(\beta, t \mid y)$ we need to define a sequence of stochastic processes $\left\{\left(X_{t}^{\langle n\rangle}, V_{t}^{\langle n\rangle}\right) ; t \geq 0\right\}_{n=1,2, \ldots}$ describing finite-velocity random motions similar to that studied in Section 3. As before, $X_{t}^{\langle n\rangle}$ and $V_{t}^{\langle n\rangle}$ respectively give the position and velocity of a particle at time $t$, with $V_{0}^{\langle n\rangle}$ identically distributed to $V_{0}$ for all $n \geq 1$. The only difference between $\left(X_{t}^{\langle n\rangle}, V_{t}^{\langle n\rangle}\right)$ and $\left(X_{t}, V_{t}\right)$ is that the random times $U_{k}^{\langle n\rangle}$ and $D_{k}^{\langle n\rangle}$ separating consecutive velocity reversals of the particle have exponential distributions with parameters

$$
\lambda_{k}^{\langle n\rangle}=\lambda(k+n-1), \quad \mu_{k}^{\langle n\rangle}=\mu(k+n-1), \quad k=1,2, \ldots ; \lambda, \mu>0,
$$

respectively, for $n=1,2, \ldots$ This implies that $X_{t}^{\langle 1\rangle}$ and $X_{t}$ are identically distributed. Let us now introduce the distribution function of the maximum of $X_{t}^{\langle n\rangle}$ :

$$
F^{\langle n\rangle}(\beta, t \mid y)=\mathrm{P}\left\{\max _{0 \leq s \leq t} X_{s}^{\langle n\rangle}<\beta \mid X_{0}^{\langle n\rangle}=0, V_{0}^{\langle n\rangle}=y\right\}, \quad n=1,2, \ldots,
$$

with $0<\beta<c t$ and $y \in\{-v, c\}$. Hereafter, we present some useful integral equations involving the distribution functions (4.2).

Proposition 4.1. For all $n \geq 1, t>0$, and $0<\beta<c t$,

$$
\begin{aligned}
F^{\langle n\rangle}(\beta, t \mid c) & \\
= & F_{U_{n}}\left(\frac{\beta}{c}\right) \bar{F}_{D_{n}}\left(\frac{c t-\beta}{c+v}\right) \\
& +\int_{0}^{\beta / c} \mathrm{~d} F_{U_{n}}(u) \int_{0}^{(c t-\beta) /(c+v)} \mathrm{d} F_{D_{n}}(\sigma) F^{\langle n+1\rangle}(\beta-c u+v \sigma, t-u-\sigma \mid c), \\
F^{\langle n\rangle}(\beta, t & \mid-v) \\
= & \bar{F}_{D_{n}}\left(\frac{c t-\beta}{c+v}\right) \\
& +\int_{0}^{(c t-\beta) /(c+v)} \mathrm{d} F_{D_{n}}(\sigma) \\
& \times \int_{0}^{(\beta+v \sigma) / c} \mathrm{~d} F_{U_{n}}(u) F^{\langle n+1\rangle}(\beta-c u+v \sigma, t-u-\sigma \mid-v) .
\end{aligned}
$$

The proof of Proposition 4.1 is similar to that of Theorem 5.1 of [2] and is thus omitted. We are now able to obtain closed-form expressions for the distribution function (4.1) in the special case $\lambda v=\mu c$.

Theorem 4.1. If $\lambda v=\mu c$, the conditional distribution functions of the maximum of $X_{t}$, for $0<\beta<c t$, are given by

$$
F(\beta, t \mid c)=\frac{1-\mathrm{e}^{-\theta \beta}}{1+\mathrm{e}^{-\theta \beta}-\mathrm{e}^{-\theta \beta} \mathrm{e}^{-\mu(c t-\beta) /(c+v)}}
$$


and

$$
\begin{aligned}
F(\beta, t \mid-v)= & \frac{2-\mathrm{e}^{-\mu(c t-\beta) /(c+v)}}{1+\mathrm{e}^{-\theta \beta}-\mathrm{e}^{-\theta \beta} \mathrm{e}^{-\mu(c t-\beta) /(c+v)}} \\
& +\mathrm{e}^{\theta \beta}\left[\theta \beta-\log \left(1+\mathrm{e}^{\theta \beta}-\mathrm{e}^{-\mu(c t-\beta) /(c+v)}\right)\right],
\end{aligned}
$$

where $\theta$ is defined in (3.11). Moreover, for $\beta \geq c t$, we have $F(\beta, t \mid c)=F(\beta, t \mid-v)=1$.

Proof. Starting from (4.3) with $n=1$, and performing an iterative procedure, when $V_{0}=c$, we obtain

$$
\begin{aligned}
F^{\langle 1\rangle}(\beta, t & \mid c) \\
= & F_{U_{1}}\left(\frac{\beta}{c}\right) \bar{F}_{D_{1}}\left(\frac{c t-\beta}{c+v}\right) \\
& +\int_{0}^{\beta / c} \mathrm{~d} F_{U_{1}}(u) \int_{0}^{(c t-\beta) /(c+v)} F_{U_{2}}\left(\frac{\beta-c u+v \sigma}{c}\right) \bar{F}_{D_{2}}\left(\frac{c t-\beta}{c+v}-\sigma\right) \mathrm{d} F_{D_{1}}(\sigma) \\
& +\int_{0}^{\beta / c} \mathrm{~d} F_{U_{1}}(u) \int_{0}^{(c t-\beta) /(c+v)} \mathrm{d} F_{D_{1}}(\sigma) \int_{0}^{(\beta-c u+v \sigma) / c} \mathrm{~d} F_{U_{2}}(x) \\
& \times \int_{0}^{(c t-\beta) /(c+v)-\sigma} F^{\langle 3\rangle}(\beta-c(u+x)+v(\sigma+y), t-u-x-\sigma-y \mid c) \mathrm{d} F_{D_{2}}(y) .
\end{aligned}
$$

By repeating substitution of $F^{\langle n\rangle}(\cdot, \cdot \mid c)$ into the last integral of (4.7), we express $F^{\langle 1\rangle}(\beta, t \mid c)$ as a series whose $i$ th term is a $2(i-1)$-dimensional integral $(i=2,3, \ldots)$. For $\lambda v=\mu c$, recalling densities (3.2) and (3.3), by means of straightforward calculations we obtain

$$
\begin{aligned}
F^{\langle 1\rangle}(\beta, t \mid c)= & \left(1-\mathrm{e}^{-\mu \beta / v}\right) \mathrm{e}^{-\mu(c t-\beta) /(c+v)} \\
& \times \sum_{k=0}^{+\infty}\left[\left(1-\mathrm{e}^{-\mu(c t-\beta) /(c+v)}\right)\left(1-\mathrm{e}^{-\mu \beta / v} \mathrm{e}^{-\mu(c t-\beta) /(c+v)}\right)\right]^{k},
\end{aligned}
$$

so that (4.5) immediately follows, noting that $F^{\langle 1\rangle}(\beta, t \mid c)=F(\beta, t \mid c), t \geq 0$. When $V_{0}=-v$, similarly to the previous case, from (4.4) we have

$$
\begin{aligned}
F^{\langle 1\rangle}(\beta, t \mid-v)= & \mathrm{e}^{-\mu(c t-\beta) /(c+v)} \\
& +\mathrm{e}^{-\mu \beta / v-2 \mu(c t-\beta) /(c+v)}\left(1-\frac{\mu(c t-\beta)}{c+v}-\mathrm{e}^{-\mu(c t-\beta) /(c+v)}\right) \\
& +\mathrm{e}^{-\mu(c t-\beta) /(c+v)}\left(1-\mathrm{e}^{-\mu(c t-\beta) /(c+v)}\right)\left(1-\mathrm{e}^{-\mu \beta / v} \mathrm{e}^{-\mu(c t-\beta) /(c+v)}\right) \\
& +\sum_{k=2}^{+\infty} A_{k}(\beta, t),
\end{aligned}
$$

where

$$
\begin{aligned}
A_{k}(\beta, t)= & \mathrm{e}^{-\mu(c t-\beta) /(c+v)}\left(1-\mathrm{e}^{-\mu \beta / v-\mu(c t-\beta) /(c+v)}\right)^{k-1} \\
\times & \left\{\left(1-\mathrm{e}^{-\mu(c t-\beta) /(c+v)}\right)^{k}\right. \\
& \left.\quad+k \mathrm{e}^{-\mu \beta / v-\mu(c t-\beta) /(c+v)}\left[-\frac{\mu(c t-\beta)}{c+v}+\sum_{j=1}^{k-1} \frac{1}{j}\left(1-\mathrm{e}^{-\mu(c t-\beta) /(c+v)}\right)^{j}\right]\right\} .
\end{aligned}
$$

After some calculations, (4.6) finally follows. 


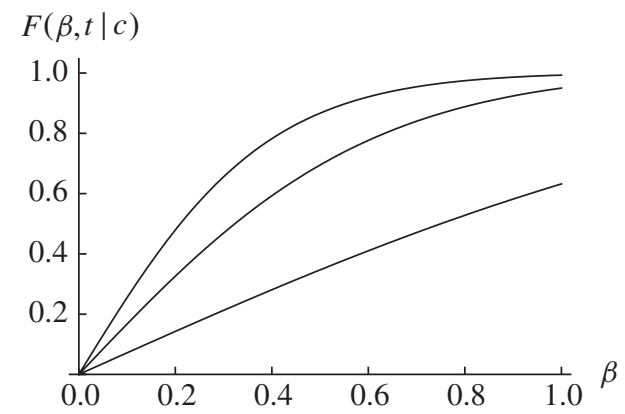

(a)

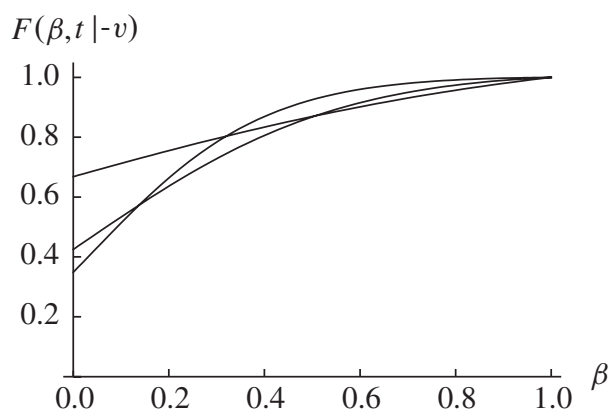

(b)

FIGURE 4: Distribution functions of the maximum of $X_{t}$ given by (a) (4.5) for $\theta=\mu=1,3,5$ (from bottom to top) and (b) (4.6) for $\theta=\mu=1,3,5$ (from top to bottom near $\beta=0$ ), with $v=c=t=1$.

Hereafter, we present the limit behavior of the distribution functions of the maximum of $X_{t}$ obtained in Theorem 4.1.

Remark 4.1. If $\lambda v=\mu c$, for any fixed $t>0$, from (4.5) and (4.6) we have

$$
\begin{gathered}
\lim _{\beta \rightarrow c t} F(\beta, t \mid c)=1-\mathrm{e}^{-\mu c t / v}, \quad \lim _{\beta \rightarrow c t} F(\beta, t \mid-v)=1, \\
\lim _{\beta \rightarrow 0} F(\beta, t \mid c)=0, \quad \lim _{\beta \rightarrow 0} F(\beta, t \mid-v)=1-\log \left(2-\mathrm{e}^{-\mu c t /(c+v)}\right) .
\end{gathered}
$$

The first limit is in agreement with (3.4). Moreover, for any fixed $\beta>0$, the following limits hold:

$$
\begin{gathered}
\lim _{t \rightarrow+\infty} F(\beta, t \mid c)=\tanh \left(\frac{\theta \beta}{2}\right) \\
\lim _{t \rightarrow+\infty} F(\beta, t \mid-v)=\frac{2}{1+\mathrm{e}^{-\theta \beta}}+\mathrm{e}^{\theta \beta}\left[\theta \beta-\log \left(1+\mathrm{e}^{\theta \beta}\right)\right],
\end{gathered}
$$

where $\theta$ is defined in (3.11).

Plots of the distribution functions obtained in Theorem 4.1 are shown in Figure 4.

\subsection{First passage time}

Let us now define the first passage time of $X_{t}$ through the boundary $\beta>0$ :

$$
T^{\beta}=\inf \left\{t \geq 0: X_{t} \geq \beta\right\} .
$$

We point out that if $V_{0}=c$ then the distribution of $T^{\beta}$ possesses a discrete component on $\beta / c$,

$$
\mathrm{P}\left\{T^{\beta}=\frac{\beta}{c} \mid V_{0}=c\right\}=\mathrm{e}^{-\lambda \beta / c},
$$

and an absolutely continuous component on $(\beta / c,+\infty)$, whose density will be denoted by $g_{\beta}(t \mid c)$. When $V_{0}=-v$, the distribution of $T^{\beta}$ is absolutely continuous on $(\beta / c,+\infty)$, with density $g_{\beta}(t \mid-v)$. Hereafter, by differentiation of (4.5) and (4.6), we obtain such densities in closed form for the special case of Theorem 4.1 . 

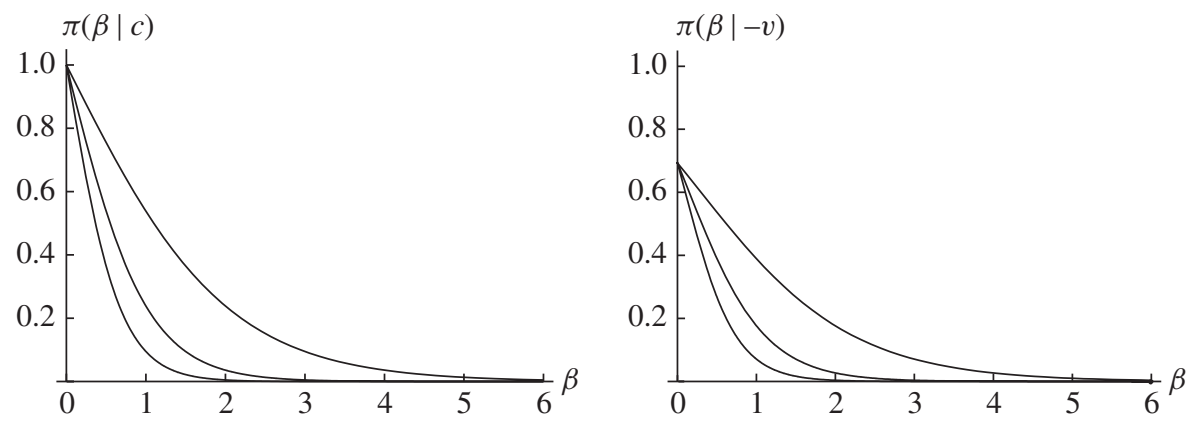

FIGURE 5: First passage time probabilities (4.13) for $\theta=1,2,3$ (from top to bottom), with initial velocity $c($ left $)$ and $-v($ right $)$.

Theorem 4.2. If $\lambda v=\mu c$, the first passage time densities of $X_{t}$ through $\beta>0$, conditional on initial velocities, are given by

$$
g_{\beta}(t \mid c)=\frac{\mu c}{c+v} \frac{\left(1-\mathrm{e}^{-\theta \beta}\right) \mathrm{e}^{-\theta \beta-\mu(c t-\beta) /(c+v)}}{\left(1+\mathrm{e}^{-\theta \beta}-\mathrm{e}^{-\theta \beta-\mu(c t-\beta) /(c+v)}\right)^{2}}, \quad t>\frac{\beta}{c},
$$

and

$$
g_{\beta}(t \mid-v)=\frac{\mu c}{c+v} \frac{\mathrm{e}^{\theta \beta-\mu(c t-\beta) /(c+v)}\left(2-\mathrm{e}^{-\mu(c t-\beta) /(c+v)}\right)}{\left(1+\mathrm{e}^{\theta \beta}-\mathrm{e}^{-\mu(c t-\beta) /(c+v)}\right)^{2}}, \quad t>\frac{\beta}{c},
$$

with $\theta$ defined in (3.11).

Let us now introduce the first crossing time probability

$$
\pi(\beta \mid y)=\mathrm{P}\left\{T^{\beta} \geq \frac{\beta}{c} \mid V_{0}=y\right\} .
$$

From (4.10)-(4.12), it is not hard to see that if $\lambda v=\mu c$ then

$$
\pi(\beta \mid y)= \begin{cases}\mathrm{e}^{-\theta \beta}\left[1+\tanh \left(\frac{\theta \beta}{2}\right)\right], & y=c, \\ \mathrm{e}^{\theta \beta}\left[\log \left(1+\mathrm{e}^{\theta \beta}\right)-\theta \beta\right]-\tanh \left(\frac{\theta \beta}{2}\right), & y=-v .\end{cases}
$$

This result is in agreement with probabilities (4.8) and (4.9) that $X_{t}$ never reaches boundary $\beta$ when $V_{0}=c$ and $V_{0}=-v$. Moreover, from (4.13) we have $\pi(0 \mid c)=1$ and $\pi(0 \mid-v)=$ $\log 2$. In conclusion, some plots of (4.13) are given in Figure 5.

\section{References}

[1] Abramowitz, M. and Stegun, I. A. (eds) (1992). Handbook of Mathematical Functions with Formulas, Graphs, and Mathematical Tables. Dover, New York.

[2] Di Crescenzo, A. (2001). On random motions with velocities alternating at Erlang-distributed random times. Adv. Appl. Prob. 33, 690-701.

[3] Di Crescenzo, A. And Martinucci, B. (2007). Random motion with gamma-distributed alternating velocities in biological modeling. In Computer Aided Systems Theory - EUROCAST 2007 (Lecture Notes Comput. Sci. 4739), Springer, Berlin, pp. 163-170. 
[4] Di Crescenzo, A. And Pellerey, F. (2002). On prices' evolutions based on geometric telegrapher's process. Appl. Stoch. Models Business Industry 18, 171-184.

[5] Ferrante, P. (2009). Interloss time in $M / M / 1 / 1$ loss system. J. Appl. Math. Stoch. Analysis, 14 pp.

[6] Foong, S. K. (1992). First-passage time, maximum displacement, and Kac's solution of the telegrapher equation. Phys. Rev. A 46, R707-R710.

[7] Gupta, R. D. And Kundu, D. (2007). Generalized exponential distribution: existing results and some recent developments. J. Statist. Planning Infer. 137, 3537-3547.

[8] Iacus, S. M. (2001). Statistical analysis of the inhomogeneous telegrapher's process. Statist. Prob. Lett. 55, 83-88.

[9] Kolesnik, A. (1998). The equations of Markovian random evolution on the line. J. Appl. Prob. 35, 27-35.

[10] LaCHAL, A. (2006). Cyclic random motions in $\mathbb{R}^{d}$-space with $n$ directions. ESAIM Prob. Statist. 10, $277-316$.

[11] LI, M. (2009). A damped diffusion framework for financial modeling and closed-form maximum likelihood estimation. J. Econom. Dynamics Control 34, 132-157.

[12] Lin, G. D. And Hu, C.-Y. (2008). On characterizations of the logistic distribution. J. Statist. Planning Infer. 138, 1147-1156.

[13] Masoliver, J. (1993). Second-order dichotomous processes: damped free motion, critical behavior, and anomalous superdiffusion. Phys. Rev. E 48, 121-135.

[14] Oluyede, B. O. (2004). Inequalities and comparisons of the Cauchy, Gauss, and logistic distributions. Appl. Math. Lett. 17, 77-82.

[15] Orsingher, E. (1985). Hyperbolic equations arising in random models. Stoch. Process. Appl. 21, 93-106.

[16] Orsingher, E. (1990). Probability law, flow function, maximum distribution of wave-governed random motions and their connections with Kirchoff's laws. Stoch. Process. Appl. 34, 49-66.

[17] Orsingher, E. (1995). Motions with reflecting and absorbing barriers driven by the telegraph equation. Random Operators Stoch. Equat. 3, 9-21.

[18] Orsingher, E. AND Bassan, B. (1992). On a $2 n$-valued telegraph signal and the related integrated process. Stoch. Stoch. Reports 38, 159-173.

[19] Pogorui, A. A. And Rodríguez-Dagnino, R. M. (2005). One-dimensional semi-Markov evolutions with general Erlang sojourn times. Random Operators Stoch. Equat. 13, 399-405.

[20] Pyke, R. (1965). Spacings. (With discussion.) J. R. Statist. Soc. B 27, 395-449.

[21] Ratanov, N. and Melnikov, A. (2008). On financial markets based on telegraph processes. Stochastics 80, 247-268.

[22] Stadje, W. and Zacks, S. (2004). Telegraph processes with random velocities. J. Appl. Prob. 41, $665-678$.

[23] Van Lieshout, M. N. M. (2006). Maximum likelihood estimation for random sequential adsorption. Adv. Appl. Prob. 38, 889-898.

[24] ZACKS, S. (2004). Generalized integrated telegraph processes and the distribution of related stopping times. J. Appl. Prob. 41, 497-507. 